\title{
Philippe Jaccottet: poésie et altérité, dir. M. FINCK et P. WERLY
}

\section{Sara Arena}

\section{(2) OpenEdition \\ Journals}

\section{Edizione digitale}

URL: https://journals.openedition.org/studifrancesi/32263

DOI: 10.4000/studifrancesi.32263

ISSN: 2421-5856

\section{Editore}

Rosenberg \& Sellier

\section{Edizione cartacea}

Data di pubblicazione: 1 août 2020

Paginazione: 441-442

ISSN: 0039-2944

Notizia bibliografica digitale

Sara Arena, «Philippe Jaccottet: poésie et altérité, dir. M. FINCK et P. wERLY», Studi Francesi [Online], 191 (LXIV | II) | 2020, online dal 01 septembre 2020, consultato il 18 septembre 2021. URL: http:// journals.openedition.org/studifrancesi/32263 ; DOI: https://doi.org/10.4000/studifrancesi.32263

Questo documento è stato generato automaticamente il 18 septembre 2021.

\section{(c) (†)}

Studi Francesi è distribuita con Licenza Creative Commons Attribuzione - Non commerciale - Non opere derivate 4.0 Internazionale. 


\title{
Philippe Jaccottet: poésie et altérité, dir. M. FINCK et P. WERLY
}

\author{
Sara Arena
}

\section{NOTIZIA}

Philippe Jaccottet: poésie et altérité, dir. M. FINCK et P. WERLY, Strasbourg, Presses

Universitaires de Strasbourg, 2018, 447 pp.

1 Nel ricco volume curato da Michèle Finck e Patrick Werly il valore dei contributi raccolti è illuminato dalla bellezza dell'opera di Philippe Jaccottet, che traluce nelle numerose citazioni d'appoggio lungo l'intera pubblicazione.

2 La particolare prospettiva dalla quale l'opera viene indagata è quella del rapporto con l'alterità, termine volutamente considerato nel più ampio ventaglio delle sue possibili accezioni, che arrivano a comprendere anche la nozione della morte. Come dichiarato nell'Avant-propos dei due curatori, il presupposto è che la scrittura del poeta, apparentemente gesto di ripiegamento su di sé, sia in realtà «le négatif d'un mouvement plus profond tourné vers l'autre» (pp. 8-9). Il complesso movimento di tensione verso l'altro è così indagato nell'opera di Jaccottet con l'obiettivo di dimostrare che esso prevale di fatto sul pur necessario «principe de solitude» (p. 9).

Dopo un corposo testo introduttivo di Michèle FINCK (Poésie, obstination d'un ton tendue vers l'autre, pp. 17-46), incentrato in particolare sulla presenza in Jaccottet di una «obstination» legata a un «ton» che cerca e presuppone l'altro, nella prima parte del libro, «Approches de l'altérité», l'alterità è intesa in senso etico, per l'appunto come rapporto con l'altro.

4 Patrick WERLY (La levée du moi et l'ouverture d'autres chemins chez Philippe Jaccottet, pp. 50-63) indaga così nelle raccolte precedenti Airs la consapevolezza di un necessario distacco dall'io, che consenta un incontro col mondo esterno. Fabio scoтto (Nommer, écouter l'Autre: métapoétique du fragment chez Philippe Jaccottet, pp. 65-76) inserisce il tema nella dimensione poetica e metapoetica del frammento, suggerendo che la scelta 
dell'«inachevé» (p. 67) permette al poeta di aderire all'istante, in una dinamica oscillatoria tra l'immediatezza del sensibile e l'intensità o la sospensione del pensiero (p. 69). Prolungando una suggestione già presente nel saggio di Fabio Scotto, Irène GAYRAUD analizza il tema dell'ascolto come espressione di apertura all'altro e alle voci del mondo, con numerosi riferimenti all'opera di Rilke («Écoute donc encore»: l'écoute comme ouverture vers l'altérité dans l'œuvre de Philippe Jaccottet, pp. 77-96). È incentrata su un solo breve testo, Obituaire, la lettura di Françoise ROUfFiAT ( $L a$ figure de l'autre dans le livre de deuil: une lecture d"'Obituaire", pp. 96-107), che prende in esame il tema della morte come estrema forma di alterità, particolarmente pressante per il poeta. Per Richard STAMELMAN (L'ombre nomade de l'autre, pp. 109-126) l'altro è tutto ciò che è «insaisissable», che resta misterioso e indecifrabile e pur si pone costantemente al soggetto nel corso dell'esistenza. In questa prospettiva le figure "assenti" di Jaccottet non sono in realtà tali: esse sono presenti, ma in quanto figure indefinibili, «effacées» (p. 116).

Nella seconda parte, «Paysages et lieux, accès à une altérité», l'alterità è considerata in una prospettiva estetica o percettiva. Laetitia REIBAUD («Lorsque nous parlerons avec la voix du rossignol...»: désir d'altérité, crainte de l'altération dans la poésie de Philippe Jaccottet, pp. 129-149) considera il costante rimando, nell'opera del poeta, allo spettacolo del mondo nella sua continua metamorfosi, tra opacità e trasparenza. La necessità di un oblio di sé per un'apertura al mondo viene collegata all'immagine degli uccelli. Per Ning YUAN (Philippe Jaccottet et «l'autre monde»: vers une pensée du cosmos, pp. 151-167) l'opera del poeta va letta alla luce del suo rapporto coi luoghi della sua esistenza e in particolare della sua ricerca - nel caos del mondo umano - di un ordine, in cui ci si possa sentire «en rapport» «avec un centre» (p. 154), che a sua volta apre a un «autre monde» (p. 167). Isabelle LEBRAT (Le dialogue avec «l'Orient limpide», nourrissante altérité: lecture de "Couleur de terre", pp. 169-184) indaga la presenza dell'Oriente in uno degli ultimi testi del poeta, Couleur de terre, concentrandosi in particolare sui significati del paesaggio come forma di alterità e luogo di rivelazione; Mathieu HILFIGER (Jaccottet: la poésie comme exigence éthique, pp. 185-209) indica come cifra della poesia di Jaccottet il bisogno imperioso di «entrer en contact avec l'immédiateté du réel» (p. 186), che fa dello sguardo sul paesaggio un gesto di empatia. Sébastien LABRUSSE sottolinea come nel paesaggio Jaccottet cerchi soprattutto la parte d'invisibile in esso contenuta, segno non dell'«irréel», ma di un «réel différent» (p. 213) e osserva come la sua opera sia in realtà lontana da una ingenua celebrazione della natura (L'humanité du paysage, pp. 211-229). Per Isabelle BALADINE HOWALD la presenza è ciò che si può cogliere tra due bagliori, un «miroitement» appena percepibile, che rende l'opera del poeta al contempo fragile e potente (Poétique du miroitement, pp. 231-234).

6 La terza parte, «Interlocuteurs (1): poètes français et étrangers», indaga il rapporto tra Jaccottet e altri autori (Laurent MOUREY, La poésie en ses dangers: Jaccottet au miroir de ses lectures (Mallarmé, Bonnefoy, Deguy), pp. 237-262); autori presenti in filigrana in singole opere (Christine DUPOUY, La venue de l'autre dans l'écriture: une lecture de la première "Semaison", pp. 263-274); in alcuni casi mediati dall'esperienza della traduzione (JeanMarc sourdillon, Poésie et mystique, l'amour de loin. Philippe Jaccottet et Jean de la Croix, pp. 275-292; Marie-Claire zIMMERMAnN, Philippe Jaccottet, traducteur de Góngora, pp. 293-308), nonché da un rapporto di conoscenza e scambio personale, come nel caso di Ungaretti (Sophie GUERMÈs, Ungaretti et Jaccottet, à la recherche du chant, pp. 309-325). Tatiana VICTOROFF ripercorre il duraturo interesse del poeta per autori e artisti russi («Faire 
passer): la Russie visible et invisible de Philippe Jaccottet (Après beaucoup d'années), pp. 327-348), mentre Yves LECLAIR approfondisce il legame con il pensiero e la poesia orientali (La sente du bout du monde de Philippe Jaccottet, pp. 349-363).

7 Nella quarta parte, «Interlocuteurs (2): peintres et musiciens», lo studio è allargato al dialogo tra Jaccottet e artisti e musicisti. Martine CRÉAC'H ripercorre i principali riferimenti del poeta in campo figurativo suggerendo tuttavia come l'apparente prossimità dell'autore sia in realtà un modo per mantenere la distanza da tali opere e «suggérer leur étrangeté» (p. 368) (Dépayser la peinture, pp. 367-378); François LALLIER analizza la visione dell'opera di Morandi, anche attraverso riferimenti ad altri autori significativi, come Leopardi (Un pèlerinage immobile (Philippe Jaccottet et Morandi), pp. 379-396); Natacha LAFond (Sur la voix/e de l'Autre: Pétrarque, Monteverdi, Purcell, pp. 397-411) e Thomas LE COLLETER («D'autres vols, plus blancs encore». Philippe Jaccottet et l'altérité musicale, pp. 413-433) si dedicano alla presenza della musica nell'opera di Jaccottet.

8 In conclusione, è presentata una lettura di Oiseaux, fleurs et fruits, importante suite della raccolta Airs, alla luce delle varianti genetiche rivelate dai manoscritti, in cui gradualmente «un sujet [...] s'émancipe de lui-même et [...] se rouvre au monde» (p. 440) (José-Flore TAPPY, L'altérité retrouvée dans "Oiseaux, fleurs et fruits" (“Airs", 1967), pp. 437-447). 